\title{
Impact of breast cancer risk factors on clinically relevant prognostic biomarkers for primary breast cancer
}

\author{
Mustapha Abubakar ${ }^{1}$ Changyuan Guo ${ }^{2} \cdot \mathrm{Hela} \mathrm{Koka}^{1} \cdot$ Bin Zhu ${ }^{1} \cdot$ Joseph Deng ${ }^{1} \cdot \mathrm{Nan} \mathrm{Hu}^{1} \cdot$ Bin Zhou ${ }^{2}$. \\ Montserrat Garcia-Closas ${ }^{1} \cdot$ Ning L $^{2} \cdot$ Xiaohong R. Yang $^{1}[$ (D)
}

Received: 11 January 2021 / Accepted: 12 June 2021 / Published online: 29 June 2021

(c) This is a U.S. government work and not under copyright protection in the U.S.; foreign copyright protection may apply 2021

\begin{abstract}
Purpose In addition to impacting incidence, risk factors for breast cancer may also influence recurrence and survival from the disease. However, it is unclear how these factors affect combinatorial biomarkers for aiding treatment decision-making in breast cancer.

Methods Patients were 8179 women with histologically confirmed invasive breast cancer, diagnosed and treated in a large cancer hospital in Beijing, China. Individual clinicopathological (tumor size, grade, lymph nodes) and immunohistochemical (IHC: ER, PR, HER2, KI67) markers were used to define clinically relevant combinatorial prognostic biomarkers, including the Nottingham Prognostic Index (NPI: combining size, grade, nodes) and IHC4 score (combining ER, PR, HER2, KI67). Odds ratios (ORs) and 95\% confidence intervals (CIs) for associations between breast cancer risk factors and quartiles (Q1Q4) of NPI and IHC4 were assessed in multivariable polytomous logistic regression models.

Results Overall, increasing parity $\left(\mathrm{OR}_{\text {trend }}(95 \% \mathrm{CI})=1.20(1.05-1.37) ; P_{\text {trend }}=0.007\right)$, overweight $(\mathrm{OR}(95 \%$ $\left.\mathrm{CI})_{\mathrm{vs} \mathrm{normal}}=1.60(1.29-1.98)\right)$, and obesity $\left(\mathrm{OR}(95 \% \mathrm{CI})_{\mathrm{vs} \mathrm{normal}}=2.12(1.43-3.14)\right)$ were associated with higher likelihood of developing tumors with high (Q4) versus low (Q1) NPI score. Conversely, increasing age $\left(\mathrm{OR}_{\text {trend }}(95 \% \mathrm{CI})=0.75(0.66-0.84)\right.$; $\left.P_{\text {trend }}<0.001\right)$ and positive family history of breast cancer $(\mathrm{FHBC})(\mathrm{OR}(95 \% \mathrm{CI})=0.66(0.45-0.95))$ were inversely associated with NPI. Only body mass index (BMI) was associated with IHC4, with overweight $(\mathrm{OR}(95 \% \mathrm{CI})$ vs normal $=0.82(0.66-1.02))$ and obese $(\mathrm{OR}(95 \% \mathrm{CI})$ vs normal $=0.52(0.36-0.76))$ women less likely to develop high IHC4 tumors. Notably, elevated BMI was associated with higher NPI irrespective of hormone receptor-expression status.

Conclusions Our findings indicate that factors affecting breast cancer incidence, particularly age, parity, FHBC, and BMI, may impact clinically relevant prognostic biomarkers with implications for surveillance, prognostication, and counseling.
\end{abstract}

Keywords Age $\cdot$ Parity $\cdot$ Overweight $\cdot$ Obese $\cdot$ Family history $\cdot$ Breast cancer $\cdot$ Prognosis $\cdot$ NPI $\cdot$ IHC4

These authors Mustapha Abubakar, Changyuan Guo, Xiaohong R. Yang, and Ning Lu contributed equally to this work.

Ning Lu

nlu03@126.com

$\triangle$ Xiaohong R. Yang royang@mail.nih.gov

1 Division of Cancer Epidemiology and Genetics, National Cancer Institute, National Institutes of Health (NIH), Bethesda, USA

2 National Cancer Center/Cancer Hospital, Chinese Academy of Medical Sciences and Peking Union Medical College, Beijing 100021, China

\begin{tabular}{ll}
\multicolumn{2}{l}{ Abbreviations } \\
BMI & $\begin{array}{l}\text { Body mass index } \\
\text { CHCAMS }\end{array}$ \\
$\begin{array}{ll}\text { Cancer Hospital, Chinese Academy of Medi- } \\
\text { cal Sciences }\end{array}$ \\
ER & $\begin{array}{l}\text { Estrogen receptor } \\
\text { FHBC }\end{array}$ \\
Family history of breast cancer \\
HR & Hormone receptor-positive \\
HR- & Hormone receptor-negative \\
IHC & Immunohistochemical/immunohistochemistry \\
OR & Odds ratio \\
PBCS & Polish Breast Cancer Study \\
PR & Progesterone receptor \\
NPI & Nottingham Prognostic Index
\end{tabular}




\section{Background}

Breast cancer is a clinically heterogeneous disease and differences in tumor behavior can influence treatment recommendations and clinical outcomes, including recurrence and survival, among breast cancer patients [1, 2]. In addition to influencing breast cancer incidence, risk factors such as elevated body mass index (BMI), nulliparity, lack of breastfeeding, and older age at first full-term pregnancy may also influence clinical outcomes following breast cancer diagnosis [3-8]. However, it is unclear whether these factors affect combinatorial biomarkers of breast cancer prognosis, which aid to guide treatment selection and patient management.

In general, most previous studies examining the relationships between breast cancer risk factors and tumor behavior have focused on the individual tumor characteristics, and not their constellation. Higher BMI, for instance, is reportedly associated with higher grade or larger size tumors; nulliparity and menopausal hormone therapy use (MHT) with highly proliferating and lobular carcinomas, respectively; and higher parity with P53 expressing tumors [9-12]. The impact of these factors on prognostic biomarkers that combine several tumor characteristics to infer tumor aggressiveness and aid treatment recommendation is, however, less well-studied.

To aid prognostication in breast cancer, several tumor characteristics including histologic grade, tumor size, lymph nodal involvement, estrogen receptor (ER), progesterone receptor (PR), human epidermal growth factor receptor 2 (HER2) and KI67, a marker of proliferation, have been combined into clinically useful prognostic algorithms [13-16]. The Nottingham prognostic index (NPI) is popular for combining information on tumor size, histologic grade, and lymph nodal involvement into a single quantitative measure [13]. The IHC4 score, on the other hand, combines information on ER, PR, HER2, and KI67 into a prognostic algorithm [16].

Despite their prognostic relevance, it remains unclear whether breast cancer risk factors influence these combinatorial prognostic markers. Our main aim in this study was, therefore, to investigate the associations between breast cancer risk factors and breast tumor behavior defined by NPI and IHC4 score among a cohort of Chinese breast cancer patients. Key findings from the primary analysis were then re-evaluated in an independent cohort of Polish patients.

\section{Methods and materials}

\section{Study population}

The main study population comprised Chinese breast cancer patients from a hospital-based case series who had histologically confirmed invasive breast cancer that were diagnosed and treated at the Cancer Hospital, Chinese Academy of Medical Sciences (CHCAMS), Beijing, China. Overall, a total of 8616 patients, aged 29-97 years at diagnosis, were recruited from CHCAMS between 2011 and 2016. Of these, 437 patients did not have complete data on hormone receptor status, i.e. ER and/or PR and hence were excluded from the analysis. This study received ethical approval from the CHCAMS Ethics Committee. Owing to the fact that this analysis did not involve interaction with human subjects or the use of individual's personal identifying information, it was granted exemption from review by the Office of Human Research Protections at the National Institutes of Health, NIH (exempt number 11751). The Polish Breast Cancer Study (PBCS) is a population-based study in Poland that enrolled women 20-74 years with histologically or cytologically confirmed breast cancer $(n=2386)$ at five participating hospitals in Warsaw and Lodz over a three-year period between 2000 and 2003 [17]. For the current analysis, we identified 972 patients from the PBCS study with complete information to allow the generation of the IHC4 score and/or NPI. Ethical approvals for PBCS were obtained from local ethics committees and all participants provided written informed consent as required by local institutional and National Cancer Institute/NIH review boards.

\section{Data on tumor clinicopathological features and breast cancer risk factors}

Data on tumor clinicopathological features, including morphology, histologic grade, tumor size, lymph nodal involvement, ER, PR, HER2, and KI67 were obtained from pathology records. Data on breast cancer risk factors, including age, BMI, family history of breast cancer (FHBC) in a first degree relative, as well as reproductive factors, including age at menarche, parity and number of children, and breastfeeding were extracted from patients' medical records. These data were collected from patients and entered into the medical records as part of patients' medical workup. Anthropometric measures, including height and weight, were obtained by trained members of staff during clinical workup. 


\section{Immunohistochemical staining}

Details of IHC staining and scoring procedures have been previously described [18]. In brief, all IHC markers were stained using standard laboratory procedures. ER, PR, and HER2 were stained using Roche rabbit monoclonal antibodies, SP1, 1E2, and 4B5 clones, at 1:1000, 1:1000, and 1:66 dilutions, respectively. KI67 was stained using mouse monoclonal antibody MIB1 based on the manufacturer optimized concentration. Staining was performed using the Roche Ventana XT autostainer for all markers. Scoring was performed by pathologists with expertise in breast cancer. Based on international conventions [2], ER and PR positivity were defined as $>1 \%$ positively staining cells while for HER2, $3+$ on IHC or amplification on fluorescent in situ hybridization (FISH) was considered positive. In keeping with results from a previous metaanalysis showing KI67 score of $25 \%$ to provide the best survival discrimination [19], we dichotomized KI67 at this cutoff-point with scores of $>25 \%$ designating high KI67 expression. Binary categories of KI67 were used in combination with standard clinical categories of ER, PR, and HER2 to define breast cancer subtypes (luminal A-like, luminal B-like, HER2-enriched, and triple negative breast cancer [TNBC]) according to internationally recommended guidelines [20]. Continuous measures of ER, PR, and KI67 were used in combination with HER2 for the calculation of the IHC4 score in keeping with the validated algorithm [16].

\section{Computation of the NPI and IHC4 score}

We calculated the NPI based on the published equation combining tumor size, grade, and nodal involvement [13]:

NPI $=0.2 \times$ tumor size $(\mathrm{cm})+$ grade + nodal status $(\mathrm{pN} 0$ $=1, \mathrm{pN} 1-3=2, \mathrm{pN} \geq 4=3$ )

We calculated the IHC4 score based on the published equation combining ER, PR, HER2 and KI67 [16]:

IHC4 score $=94.7 \times\{(-0.100 \mathrm{ER} 10)+(-0.079 \mathrm{PR} 10)$ $+(0.586 \mathrm{HER} 2)+[0.240 \ln (1+10 \times \mathrm{Ki} 67)]\}$

\section{Statistical analysis}

The following breast cancer risk factors were examined: age: $<35$ (reference), 35-45, 45-55, $>55$ years; parity: nulliparous (reference), 1, 2, $\geq 3$ children; age at menarche: $\leq 12$ (reference), $13,14, \geq 15$ years; $\mathrm{BMI}$, in $\mathrm{kg} / \mathrm{m}^{2}$ : underweight $(<18.5)$, normal (18.5-25; reference), overweight (25-30), and obese ( $>30)$; and FHBC in a first degree relative: yes (if present) or no (if absent; reference). NPI and IHC4 score were categorized into quartiles as follows; Q1 $(<25$ th percentile); Q2 (25th-50th percentile); Q3 (50th-75th percentile); and Q4 (>75th percentile). Associations between breast cancer risk factors and tumor-related prognostic indicators were assessed in polytomous logistic regression models with quartiles of NPI and IHC4 score as outcomes (Q1 = baseline category) and breast cancer risk factors as predictors. Analyses were performed overall and following stratification by age ( $\leq 50$ and $>50)$, as surrogate for menopausal status, and by tumor hormone receptor (HR) expression status (i.e. HR + and HR-). In sensitivity analysis, we used PREDICT[14], another combinatorial prognostic marker that contains tumor size, grade, and lymph nodal involvement, in place of the NPI and assessed relationships with breast cancer risk factors. To assess which if any, of the individual clinicopathologic (tumor size, histologic grade, nodal involvement) and IHC (ER, PR, HER2 and KI67) factors was driving associations between risk factors and these combinatorial prognostic factors, we modeled relevant breast cancer risk factors as outcome variables and mutually adjusted for the individual clinicopathological and IHC features as predictors. The contribution of individual factors to model prediction was determined by assessing change in likelihood ratio Chi-square $\left(\operatorname{LR} \chi^{2}\right)$ when the factor was removed from the fully adjusted model. For those factors that were significantly related to NPI and/or IHC4 in the Chinese population, we repeated the analysis in an independent sample of 972 breast cancer cases from PBCS [17]. Missing values on risk factor covariates were addressed by the listwise deletion approach. In additional sensitivity analyses, we created indicators for missing values on the covariates that were included in multivariable models. All analyses were two-sided and performed using Stata statistical software version 16.1 .

\section{Results}

\section{Distribution of baseline clinicopathological factors and breast cancer risk factors among study participants}

Table 1 describes the distribution of clinicopathologic as well as lifestyle and reproductive factors in this dataset consisting of 8,179 breast cancer patients, overall and stratified by HR status. The mean age at diagnosis was 51.8 years, which did not differ significantly by HR status. The majority of these patients had intermediate or high grade (91\%) tumors. Similarly, node negative (53\%), ER + (77\%), PR + (76\%), HER2- (80\%), and low KI67 (55\%) tumors predominated. Notably, the frequencies of all clinicopathologic characteristics differed significantly by HR status, with HR- tumors having higher frequencies of aggressive tumor 
Table 1 Distributions of clinicopathological and breast cancer risk factors, overall and by tumor hormone receptor expression status, among Chinese breast cancer patients

\begin{tabular}{|c|c|c|c|c|c|c|c|}
\hline \multirow[t]{2}{*}{ Characteristic } & \multicolumn{2}{|c|}{ Overall $(n=8179)$} & \multicolumn{2}{|c|}{$\mathrm{HR}+(n=6675)$} & \multicolumn{2}{|c|}{$\mathrm{HR}-(n=1504)$} & \multirow[t]{2}{*}{$P$ value } \\
\hline & Freq & $\%$ & Freq & $\%$ & Freq & $\%$ & \\
\hline \multicolumn{8}{|l|}{ Age, years } \\
\hline$<35$ & 454 & 5.3 & 347 & 5.2 & 75 & 5.0 & \\
\hline $35-45$ & 2095 & 24.3 & 1640 & 24.5 & 335 & 22.3 & \\
\hline $45-55$ & 2982 & 34.7 & 2301 & 34.5 & 544 & 36.1 & \\
\hline$>55$ & 3068 & 35.7 & 2387 & 35.8 & 550 & 36.6 & 0.26 \\
\hline \multicolumn{8}{|c|}{ Histologic grade } \\
\hline Low & 670 & 8.6 & 624 & 10.2 & 11 & 0.8 & \\
\hline Intermediate & 4655 & 59.9 & 4051 & 66.1 & 412 & 30.6 & \\
\hline High & 2450 & 31.5 & 1455 & 23.7 & 923 & 68.6 & $<0.001$ \\
\hline \multicolumn{8}{|l|}{ Size } \\
\hline$\leq 2 \mathrm{~cm}$ & 3531 & 47.3 & 2799 & 48.0 & 540 & 40.6 & \\
\hline$>2 \mathrm{~cm}$ & 3939 & 52.7 & 3027 & 52.0 & 791 & 59.4 & $<0.001$ \\
\hline \multicolumn{8}{|c|}{ Nodal involvement } \\
\hline None & 3798 & 53.0 & 2968 & 52.2 & 713 & 57.1 & \\
\hline 1 & 985 & 13.8 & 795 & 14.0 & 153 & 12.3 & \\
\hline 2 & 563 & 7.9 & 469 & 8.3 & 79 & 6.3 & \\
\hline$\geq 3$ & 1806 & 25.3 & 1451 & 25.5 & 303 & 24.3 & 0.006 \\
\hline \multicolumn{8}{|l|}{ ER } \\
\hline Negative & 1897 & 23.2 & & & & & \\
\hline Positive & 6285 & 76.8 & & & & & \\
\hline \multicolumn{8}{|l|}{ PR } \\
\hline Negative & 1952 & 23.9 & & & & & \\
\hline Positive & 6221 & 76.1 & & & & & \\
\hline \multicolumn{8}{|l|}{ HER2 } \\
\hline Negative & 6556 & 80.3 & 5627 & 84.5 & 926 & 61.8 & \\
\hline Positive & 1604 & 19.7 & 1031 & 15.5 & 572 & 38.2 & $<0.001$ \\
\hline \multicolumn{8}{|l|}{ KI67 } \\
\hline Low & 4432 & 55.2 & 4066 & 62.0 & 356 & 24.5 & \\
\hline High & 3598 & 44.8 & 2494 & 38.0 & 1098 & 75.5 & $<0.001$ \\
\hline \multicolumn{8}{|c|}{ Age at Menarche, years } \\
\hline$\leq 12$ & 730 & 10.9 & 596 & 11.2 & 106 & 9.1 & \\
\hline 13 & 1248 & 18.6 & 982 & 18.4 & 235 & 20.2 & \\
\hline 14 & 1509 & 22.6 & 1197 & 22.4 & 354 & 21.9 & \\
\hline$\geq 15$ & 3207 & 47.9 & 2560 & 48.0 & 566 & 48.8 & 0.13 \\
\hline \multicolumn{8}{|l|}{ Parity } \\
\hline Nulliparous & 304 & 3.5 & 241 & 3.6 & 45 & 3.0 & \\
\hline 1 & 3985 & 46.3 & 3186 & 47.7 & 676 & 45.0 & \\
\hline 2 & 1920 & 22.3 & 1497 & 22.4 & 354 & 23.5 & \\
\hline$\geq 3$ & 2407 & 27.9 & 1751 & 26.3 & 429 & 28.5 & 0.09 \\
\hline \multicolumn{8}{|l|}{ Breastfeeding } \\
\hline Never & 733 & 12.5 & 584 & 12.6 & 111 & 10.5 & \\
\hline Ever & 5142 & 87.5 & 4045 & 87.4 & 945 & 89.5 & 0.06 \\
\hline \multicolumn{8}{|c|}{ Body mass index (BMI) } \\
\hline Underweight & 155 & 2.1 & 121 & 2.1 & 25 & 2.0 & \\
\hline Normal & 3946 & 54.2 & 3090 & 53.7 & 703 & 55.4 & \\
\hline Overweight & 2586 & 35.5 & 2063 & 35.9 & 440 & 34.6 & \\
\hline Obese & 595 & 8.2 & 480 & 8.3 & 102 & 8.0 & 0.76 \\
\hline \multicolumn{8}{|l|}{ Family history } \\
\hline Absent & 6721 & 92.4 & 5316 & 92.4 & 1180 & 92.8 & \\
\hline
\end{tabular}


Table 1 (continued)

\begin{tabular}{|c|c|c|c|c|c|c|c|}
\hline \multirow[t]{2}{*}{ Characteristic } & \multicolumn{2}{|c|}{ Overall $(n=8179)$} & \multicolumn{2}{|c|}{$\mathrm{HR}+(n=6675)$} & \multicolumn{2}{|c|}{$\mathrm{HR}-(n=1504)$} & \multirow[t]{2}{*}{ Pvalue } \\
\hline & Freq & $\%$ & Freq & $\%$ & Freq & $\%$ & \\
\hline Present & 554 & 7.6 & 440 & 7.6 & 91 & 7.2 & 0.55 \\
\hline
\end{tabular}

characteristics than HR + tumors. In terms of breast cancer risk factors, the majority of the patients had at least one child (97\%), had breastfed (87\%), had normal BMI (54\%), and had a negative FHBC in a first degree relative (92\%), none of which varied by HR status (Table 1). In general, patients from the PBCS study were older, experienced menarche earlier, and were more frequently nulliparous or obese than patients from the CHCAMS study (Supplementary Table 1).

\section{Associations between breast cancer risk factors and levels of Nottingham prognostic index (NPI)}

Table 2 shows the associations between breast cancer risk factors and the NPI. We found increasing age to be statistically significantly inversely associated with higher NPI $\left(\mathrm{OR}_{\text {trend }}(95 \% \mathrm{CI})=0.75(0.66-0.84), \mathrm{P}_{\text {trend }}<0.001\right)$. Conversely, higher parity was significantly associated with higher NPI $\left(\mathrm{OR}_{\text {trend }}(95 \% \mathrm{CI})=1.20(1.05-1.37)\right.$, $\left.\mathrm{P}_{\text {trend }}=0.007\right)$. Furthermore, overweight (OR $(95 \%$ $\mathrm{CI})=1.60(1.29-1.98))$ and obese $(\mathrm{OR}(95 \% \mathrm{CI})=2.12$ (1.43-3.14) women were substantially more likely than normal weight women to have high (Q4 vs Q1) NPI values. There was a significant trend with increasing BMI and $\mathrm{NPI}\left(\mathrm{OR}_{\text {trend }}(95 \% \mathrm{CI})=1.53(1.30-1.79), \mathrm{P}_{\text {trend }}<0.001\right) . \mathrm{A}$ positive $\mathrm{FHBC}$ in a first degree relative was inversely associated with higher NPI values (OR $(95 \% \mathrm{CI})$ Q4 vs Q1 $=0.66$ $(0.45-0.95) ; P=0.027)$. Other factors, including breastfeeding and age at menarche were not associated with the NPI. In sensitivity analysis using PREDICT, we found strikingly similar associations between age, parity, BMI, and family history with PREDICT as we did in relation to the NPI (Supplementary Table 2). The associations between parity, BMI, and FHBC with NPI were similar in women with $\mathrm{HR}+$ and HR- breast cancer (Table 3), in younger and older women (Supplementary Table 3), and in analysis accounting for missing values on covariates (Supplementary Table 4).

\section{Associations between breast cancer risk factors and levels of immunohistochemical 4 (IHC4) score}

Of the risk factors assessed, only BMI demonstrated significant associations with IHC4 score. In contrast to the positive associations that we found between elevated BMI and high NPI, elevated BMI was inversely associated with the IHC4 score. In comparison to normal weight women, overweight $(\mathrm{OR}(95 \% \mathrm{CI})=0.82(0.66-1.02))$ and obese $(\mathrm{OR}$
$(95 \% \mathrm{CI})=0.52(0.36-0.76))$ women were less likely to have tumors with high IHC4 score (Table 4). In stratified analysis by age, we found the inverse association between increasing BMI and the IHC4 to be statistically significantly stronger among older than younger women ( $\mathrm{P}$ value for heterogeneity $[\mathrm{P}$-het $]=0.006$ ) but estimates were in the same direction (Supplementary Table 5). We did not find evidence for associations between age at menarche, parity, and breastfeeding with the IHC4. Overall, the results were similar in analysis accounting for missing values on covariates (Supplementary Table 6).

\section{Age at onset, parity, BMI and FHBC in relation to NPI} and IHC4 score among Polish breast cancer patients

To check whether the associations we found between age at onset, parity, BMI, and family history with clinical prognostic markers were seen in other populations, we used data from a subset of 972 Polish breast cancer patients. Similar to the findings among Chinese patients, increasing age and a positive FHBC in a first degree relative were associated with lower NPI values even though the estimates did not attain statistical significance. In addition, overweight (OR $(95 \% \mathrm{CI})=1.34(0.82-2.19)$ and obese $(\mathrm{OR}(95 \% \mathrm{CI})=2.27$ (1.32-3.89) women were more likely to have high NPI when compared to normal weight women (Table 5), which is similar to what we found among the Chinese women. None of the factors evaluated was statistically significantly associated with the IHC4 score among Polish women overall. However, consistent with the findings among Chinese women, the inverse association between elevated BMI and levels of IHC4 score were stronger among older than younger women (P-het $=0.04$; Supplementary Table 7).

\section{Associations between parity, BMI, and family history with individual clinicopathologic characteristics}

In analysis to determine the contributions of individual clinicopathologic factors to predictive models for BMI, parity, and family history in relation to clinical prognostic markers, we modeled each of these risk factors as an outcome variable and clinicopathological and IHC factors as explanatory variables. In stepwise analyses, we removed each clinicopathologic factor from the full model and assessed the change in likelihood ration Chi-square $\left(\Delta \mathrm{LR} \chi^{2}\right)$, which was compared with the nested model 
Table 2 Odds ratios (ORs) and 95\% confidence intervals (CIs) for the associations between breast cancer risk factors and levels of the Nottingham prognostic index (NPI) among Chinese breast cancer patients with complete data $(n=3668)$

\begin{tabular}{|c|c|c|c|c|c|c|c|}
\hline \multirow[t]{3}{*}{ Characteristic } & \multicolumn{7}{|l|}{ NPI } \\
\hline & \multirow{2}{*}{$\begin{array}{l}\text { Q1 (reference) } \\
\mathrm{N}\end{array}$} & \multicolumn{2}{|l|}{ Q2 } & \multicolumn{2}{|l|}{ Q3 } & \multicolumn{2}{|l|}{ Q4 } \\
\hline & & $\mathrm{N}$ & OR $(95 \% \mathrm{CI})$ & $\mathrm{N}$ & OR $(95 \% \mathrm{CI})$ & $\mathrm{N}$ & OR $(95 \% \mathrm{CI})$ \\
\hline \multicolumn{8}{|l|}{ Age, years } \\
\hline$<35$ & 26 & 43 & 1.00 (reference) & 56 & 1.00 (reference) & 74 & 1.00 (reference) \\
\hline $35-45$ & 211 & 229 & $0.66(0.38,1.13)$ & 234 & $0.49(0.29,0.84)$ & 208 & $0.31(0.18,0.52)$ \\
\hline $45-55$ & 322 & 326 & $0.54(0.32,0.92)$ & 315 & $0.36(0.21,0.60)$ & 294 & $0.23(0.14,0.39)$ \\
\hline$>55$ & 326 & 387 & $0.64(0.37,1.10)$ & 316 & $0.34(0.20,0.57)$ & 301 & $0.22(0.13,0.37)$ \\
\hline $\mathrm{OR}_{\text {trend }}$ & & & $0.94(0.84,1.06)$ & & $0.79(0.71,0.89)$ & & $0.75(0.66,0.84)$ \\
\hline$P$ trend & & & 0.33 & & $<0.001$ & & $<0.001$ \\
\hline \multicolumn{8}{|c|}{ Age at Menarche, years } \\
\hline$\leq 12$ & 105 & 103 & 1.00 (reference) & 98 & 1.00 (reference) & 100 & 1.00 (reference) \\
\hline 13 & 175 & 194 & $1.11(0.77,1.58)$ & 179 & $1.02(0.70,1.47)$ & 152 & $0.88(0.60,1.28)$ \\
\hline 14 & 206 & 241 & $1.18(0.83,1.66)$ & 210 & $1.04(0.73,1.48)$ & 198 & $0.98(0.68,1.40)$ \\
\hline$\geq 15$ & 399 & 447 & $1.08(0.78,1.49)$ & 434 & $1.06(0.76,1.48)$ & 427 & $1.03(0.74,1.43)$ \\
\hline $\mathrm{OR}_{\text {trend }}$ & & & $1.01(0.92,1.11)$ & & $1.02(0.93,1.13)$ & & $1.04(0.94,1.15)$ \\
\hline$P$ trend & & & 0.85 & & 0.64 & & 0.40 \\
\hline \multicolumn{8}{|l|}{ Parity } \\
\hline Nulliparous & 37 & 47 & 1.00 (reference) & 37 & 1.00 (reference) & 28 & 1.00 (reference) \\
\hline 1 & 485 & 510 & $0.83(0.52,1.34)$ & 425 & $0.95(0.57,1.59)$ & 399 & $1.29(0.74,2.23)$ \\
\hline 2 & 219 & 263 & $0.81(0.49,1.33)$ & 269 & $1.21(0.71,2.06)$ & 251 & $1.60(0.90,2.84)$ \\
\hline$\geq 3$ & 144 & 165 & $0.67(0.40,1.14)$ & 190 & $1.18(0.67,2.06)$ & 199 & $1.75(0.97,3.17)$ \\
\hline $\mathrm{OR}_{\text {trend }}$ & & & $0.91(0.80,1.04)$ & & $1.13(0.99,1.29)$ & & $1.20(1.05,1.37)$ \\
\hline$P$ trend & & & 0.17 & & 0.07 & & 0.007 \\
\hline \multicolumn{8}{|l|}{ Breastfeeding } \\
\hline Never & 103 & 106 & 1.00 (reference) & 90 & 1.00 (reference) & 89 & 1.00 (reference) \\
\hline Ever & 687 & 717 & $0.97(0.71,1.31)$ & 691 & $1.16(0.83,1.60)$ & 652 & $1.10(0.79,1.53)$ \\
\hline$P$ value & & & 0.82 & & 0.38 & & 0.55 \\
\hline \multicolumn{8}{|l|}{$B M I$} \\
\hline Underweight & 19 & 16 & $0.75(0.38,1.52)$ & 15 & $0.76(0.37,1.57)$ & 17 & $0.94(0.47,1.90)$ \\
\hline Normal & 537 & 508 & 1.00 (reference) & 474 & 1.00 (reference) & 433 & 1.00 (reference) \\
\hline Overweight & 278 & 375 & $1.47(1.20,1.81)$ & 341 & $1.46(1.18,1.81)$ & 345 & $1.60(1.29,1.98)$ \\
\hline Obese & 51 & 86 & $1.91(1.30,2.80)$ & 91 & $2.27(1.54,3.33)$ & 82 & $2.12(1.43,3.14)$ \\
\hline $\mathrm{OR}_{\text {trend }}$ & & & $1.42(1.22,1.66)$ & & $1.50(1.28,1.76)$ & & $1.53(1.30,1.79)$ \\
\hline$P$ trend & & & $<0.001$ & & $<0.001$ & & $<0.001$ \\
\hline \multicolumn{8}{|l|}{ Family history } \\
\hline Absent & 792 & 913 & 1.00 (reference) & 863 & 1.00 (reference) & 822 & 1.00 (reference) \\
\hline Present & 93 & 72 & $0.73(0.52,1.03)$ & 58 & $0.64(0.45,0.93)$ & 55 & $0.66(0.45,0.95)$ \\
\hline P value & & & 0.07 & & 0.02 & & 0.03 \\
\hline
\end{tabular}

ORs and 95\% CIs were from polytomous logistic regression models (Q1 was the base (comparison) category) with mutual adjustments for age, parity, age at menarche, body mass index (BMI), and family history. Parity was adjusted in the main model (presented in table) and this was substituted for breastfeeding in a separate model. All models were further adjusted for year of diagnosis and breast cancer subtype

using LR tests. In general, age was most relevant for the estimation of models for BMI, parity, and FHBC. This was followed by tumor size, ER, HER2 and grade for obesity; node status, grade, tumor size and ER for parity; and node status, HER2, and grade for FHBC (Table 6).

\section{Discussion}

In this large-scale analysis of over 8,000 Chinese breast cancer patients, we assessed relationships between breast 
Table 3 Odds ratios (ORs) and 95\% confidence intervals (CIs) for associations between parity, body mass index (BMI), and family history and levels of the Nottingham prognostic index (NPI) in stratified analyses by hormone receptor-expression status among Chinese breast cancer patients

\begin{tabular}{|c|c|c|c|}
\hline \multirow[t]{3}{*}{ Characteristic } & \multicolumn{3}{|l|}{ NPI score } \\
\hline & $\mathrm{Q} 2$ vs $\mathrm{Q} 1$ & $\mathrm{Q} 3$ vs Q1 & $\mathrm{Q} 4$ vs Q1 \\
\hline & OR $(95 \%$ CI $)$ & OR $(95 \% \mathrm{CI})$ & OR $(95 \% \mathrm{CI})$ \\
\hline \multicolumn{4}{|l|}{$\mathrm{HR}+$} \\
\hline \multicolumn{4}{|l|}{ Parity } \\
\hline Nulliparous & 1.00 (reference) & 1.00 (reference) & 1.00 (reference) \\
\hline 1 & $\begin{array}{c}1.01(0.61 \\
1.70)\end{array}$ & $\begin{array}{c}1.04(0.61, \\
1.76)\end{array}$ & $1.39(0.78,2.46)$ \\
\hline 2 & $\begin{array}{c}0.90(0.52 \\
1.54)\end{array}$ & $\begin{array}{c}1.24(0.72 \\
2.16)\end{array}$ & $1.71(0.94,3.11)$ \\
\hline$\geq 3$ & $\begin{array}{c}0.73(0.41 \\
1.29)\end{array}$ & $\begin{array}{c}1.18(0.66, \\
2.11)\end{array}$ & $1.74(0.94,3.25)$ \\
\hline$O R_{\text {trend }}$ & $\begin{array}{c}0.89(0.77 \\
1.02)\end{array}$ & $\begin{array}{c}1.10(0.96 \\
1.27)\end{array}$ & $1.18(1.02,1.36)$ \\
\hline$P$ trend & 0.09 & 0.18 & 0.02 \\
\hline \multicolumn{4}{|l|}{$B M I$} \\
\hline $\begin{array}{l}\text { Under- } \\
\text { weight }\end{array}$ & $\begin{array}{c}0.75(0.34 \\
1.65)\end{array}$ & $\begin{array}{c}0.98(0.47, \\
2.04)\end{array}$ & $0.94(0.45,2.00)$ \\
\hline Normal & 1.00 (reference) & 1.00 (reference) & 1.00 (reference) \\
\hline Overweight & $\begin{array}{c}1.40(1.12 \\
1.75)\end{array}$ & $\begin{array}{c}1.54(1.22, \\
1.93)\end{array}$ & $1.54(1.23,1.94)$ \\
\hline Obese & $\begin{array}{c}1.88(1.24 \\
2.83)\end{array}$ & $\begin{array}{c}2.56(1.72, \\
3.82)\end{array}$ & $2.08(1.37,3.15)$ \\
\hline$O R_{\text {trend }}$ & $\begin{array}{c}1.39(1.17 \\
1.64)\end{array}$ & $\begin{array}{c}1.59(1.34, \\
1.87)\end{array}$ & $1.49(1.26,1.77)$ \\
\hline$P$ trend & $<0.001$ & $<0.001$ & $<0.001$ \\
\hline \multicolumn{4}{|l|}{$\begin{array}{l}\text { Family his- } \\
\text { tory }\end{array}$} \\
\hline Absent & 1.00 (reference) & 1.00 (reference) & 1.00 (reference) \\
\hline Present & $\begin{array}{c}0.75(0.52 \\
1.08)\end{array}$ & $\begin{array}{c}0.58(0.39 \\
0.86)\end{array}$ & $0.64(0.43,0.95)$ \\
\hline Pvalue & 0.11 & 0.007 & 0.02 \\
\hline \multicolumn{4}{|l|}{$\mathrm{HR}-$} \\
\hline \multicolumn{4}{|l|}{ Parity } \\
\hline Nulliparous & 1.00 (reference) & 1.00 (reference) & 1.00 (reference) \\
\hline 1 & $\begin{array}{c}0.40(0.12 \\
1.32)\end{array}$ & $\begin{array}{c}1.17(0.27, \\
5.10)\end{array}$ & $0.92(0.22,3.73)$ \\
\hline 2 & $\begin{array}{c}0.48(0.14 \\
1.65)\end{array}$ & $\begin{array}{c}1.23(0.27 \\
5.59)\end{array}$ & $0.90(0.21,3.82)$ \\
\hline$\geq 3$ & $\begin{array}{c}0.60(0.16 \\
2.18)\end{array}$ & $\begin{array}{c}1.60(0.33, \\
7.74)\end{array}$ & $1.55(0.35,6.92)$ \\
\hline$O R_{\text {trend }}$ & $\begin{array}{c}1.07(0.79 \\
1.43)\end{array}$ & $\begin{array}{c}1.10(0.80, \\
1.52)\end{array}$ & $1.17(0.85,1.59)$ \\
\hline$P$ trend & 0.67 & 0.55 & 0.33 \\
\hline \multicolumn{4}{|l|}{$B M I$} \\
\hline $\begin{array}{l}\text { Under- } \\
\text { weight }\end{array}$ & $\begin{array}{c}1.22(0.19 \\
7.68)\end{array}$ & $\begin{array}{c}1.08(0.14, \\
8.07)\end{array}$ & $\begin{array}{c}2.03(0.32 \\
12.94)\end{array}$ \\
\hline Normal & 1.00 (reference) & 1.00 (reference) & 1.00 (reference) \\
\hline Overweight & $\begin{array}{c}1.10(0.69, \\
1.75)\end{array}$ & $\begin{array}{c}1.05(0.63 \\
1.73)\end{array}$ & $1.42(0.87,2.31)$ \\
\hline
\end{tabular}

Table 3 (continued)

\begin{tabular}{|c|c|c|c|}
\hline \multirow[t]{3}{*}{ Characteristic } & \multicolumn{3}{|l|}{ NPI score } \\
\hline & $\mathrm{Q} 2$ vs Q1 & $\mathrm{Q} 3$ vs Q1 & $\mathrm{Q} 4$ vs Q1 \\
\hline & OR $(95 \% \mathrm{CI})$ & OR $(95 \% \mathrm{CI})$ & OR $(95 \% \mathrm{CI})$ \\
\hline Obese & $\begin{array}{c}1.49(0.58 \\
3.83)\end{array}$ & $\begin{array}{c}1.48(0.54 \\
4.02)\end{array}$ & $2.31(0.90,5.91)$ \\
\hline$O R_{\text {trend }}$ & $\begin{array}{c}1.13(0.79 \\
1.61)\end{array}$ & $\begin{array}{c}1.13(0.77 \\
1.66)\end{array}$ & $1.46(1.02,2.10)$ \\
\hline$P$ trend & 0.49 & 0.53 & 0.04 \\
\hline \multicolumn{4}{|l|}{$\begin{array}{l}\text { Family his- } \\
\text { tory }\end{array}$} \\
\hline Absent & 1.00 (reference) & 1.00 (reference) & 1.00 (reference) \\
\hline Present & $\begin{array}{c}1.63(0.73 \\
3.64)\end{array}$ & $\begin{array}{c}0.52(0.17 \\
1.59)\end{array}$ & $1.10(0.43,2.77)$ \\
\hline Pvalue & 0.97 & 0.25 & 0.84 \\
\hline
\end{tabular}

OR and $95 \%$ CI were from polytomous logistic regression models (Q1 of NPI was the base (comparison) category) with mutual adjustments for parity, body mass index (BMI), age at menarche, and family history. Models were further adjusted for diagnosis year and breast cancer subtype

cancer risk factors and clinically important combinatorial prognostic biomarkers in breast cancer. Specifically, we used routinely available clinicopathological (tumor size, histologic grade, or nodal involvement) and IHC (ER, PR, HER2, and KI67) parameters to compute the NPI and IHC4 score and investigated relationships with breast cancer risk factors. We found younger age, higher parity, being overweight or obese, and not having a FHBC to be associated with more clinically advanced breast cancer, represented by high NPI. Unlike what we observed in relation to the NPI, however, being overweight or obese was associated with lower IHC4 score, indicating less aggressive breast cancer phenotype. Nevertheless, accounting for HR expression, women with elevated BMI were more likely to have clinically advanced HR + and HR- breast cancers. Taken together, these results suggest that breast cancer risk factors may influence the biology and clinical presentation of breast tumors, with implications for improved surveillance and the development of prognostic tools incorporating risk factors.

In addition to tumor-related factors, the age at breast cancer diagnosis has long been recognized to be associated with worse clinical outcomes [21-25]. Our age-related findings are in keeping with those from previous studies demonstrating associations between younger age at onset and more aggressive tumor characteristics even among HR + tumors [21] and suggests that previously reported associations may partly be mediated by tumor characteristics, particularly those contained in the NPI, i.e. size, grade, and lymph nodal involvement. Nevertheless, further studies incorporating mediation analysis will be required to conclusively 
Table 4 Odds ratios (ORs) and 95\% confidence intervals (CIs) for the associations between breast cancer risk factors and levels of the immunohistochemical 4 (IHC4) score among Chinese breast cancer patients with complete data $(n=3475)$

\begin{tabular}{|c|c|c|c|c|c|c|c|}
\hline \multirow[t]{3}{*}{ Characteristic } & \multicolumn{7}{|l|}{ IHC4 score } \\
\hline & \multirow{2}{*}{$\begin{array}{l}\text { Q1 (reference) } \\
\mathrm{N}\end{array}$} & \multicolumn{2}{|l|}{ Q2 } & \multicolumn{2}{|l|}{ Q3 } & \multicolumn{2}{|l|}{ Q4 } \\
\hline & & $\mathrm{N}$ & $\mathrm{OR}(95 \% \mathrm{CI})$ & $\mathrm{N}$ & $\mathrm{OR}(95 \% \mathrm{CI})$ & $\mathrm{N}$ & $\mathrm{OR}(95 \% \mathrm{CI})$ \\
\hline \multicolumn{8}{|l|}{ Age, years } \\
\hline$<35$ & 28 & 54 & 1.00 (reference) & 50 & 1.00 (reference) & 52 & 1.00 (reference) \\
\hline $35-45$ & 245 & 218 & $0.49(0.29,0.82)$ & 175 & $0.44(0.26,0.74)$ & 199 & $0.51(0.30,0.86)$ \\
\hline $45-55$ & 310 & 255 & $0.48(0.29,0.80)$ & 284 & $0.61(0.37,1.03)$ & 336 & $0.77(0.46,1.30)$ \\
\hline$>55$ & 317 & 337 & $0.64(0.38,1.06)$ & 323 & $0.68(0.41,1.15)$ & 292 & $0.67(0.40,1.14)$ \\
\hline $\mathrm{OR}_{\text {trend }}$ & & & $1.04(0.93,1.16)$ & & $1.10(0.98,1.24)$ & & $1.05(0.93,1.18)$ \\
\hline$P$ trend & & & 0.54 & & 0.09 & & 0.42 \\
\hline \multicolumn{8}{|c|}{ Age at Menarche, years } \\
\hline$\leq 12$ & 97 & 116 & 1.00 (reference) & 88 & 1.00 (reference) & 90 & 1.00 (reference) \\
\hline 13 & 179 & 141 & $0.65(0.45,0.93)$ & 171 & $1.02(0.70,1.48)$ & 177 & $1.07(0.73,1.56)$ \\
\hline 14 & 198 & 204 & $0.86(0.61,1.21)$ & 200 & $1.09(0.76,1.56)$ & 202 & $1.13(0.77,1.63)$ \\
\hline$\geq 15$ & 426 & 403 & $0.77(0.56,1.06)$ & 373 & $0.88(0.63,1.24)$ & 410 & $1.01(0.71,1.42)$ \\
\hline $\mathrm{OR}_{\text {trend }}$ & & & $0.96(0.87,1.05)$ & & $0.94(0.85,1.04)$ & & $0.99(0.90,1.10)$ \\
\hline$P$ trend & & & 0.39 & & 0.21 & & 0.91 \\
\hline \multicolumn{8}{|l|}{ Parity } \\
\hline Nulliparous & 47 & 27 & 1.00 (reference) & 29 & 1.00 (reference) & 39 & 1.00 (reference) \\
\hline 1 & 460 & 438 & $1.94(1.16,3.24)$ & 385 & $1.55(0.93,2.57)$ & 427 & $1.25(0.77,2.02)$ \\
\hline 2 & 247 & 225 & $1.70(1.00,2.90)$ & 240 & $1.60(0.94,2.72)$ & 238 & $1.11(0.66,1.85)$ \\
\hline$\geq 3$ & 146 & 174 & $2.01(1.15,3.53)$ & 178 & $1.79(1.03,3.13)$ & 175 & $1.19(0.69,2.04)$ \\
\hline $\mathrm{OR}_{\text {trend }}$ & & & $1.06(0.93,1.21)$ & & $1.11(0.97,1.26)$ & & $0.98(0.85,1.12)$ \\
\hline$P$ trend & & & 0.37 & & 0.13 & & 0.76 \\
\hline \multicolumn{8}{|l|}{ Breastfeeding } \\
\hline Never & 108 & 87 & 1.00 (reference) & 82 & 1.00 (reference) & 92 & 1.00 (reference) \\
\hline Ever & 686 & 660 & $1.20(0.88,1.65)$ & 640 & $1.26(0.91,1.74)$ & 679 & $1.17(0.84,1.62)$ \\
\hline$P$ value & & & 0.25 & & 0.16 & & 0.36 \\
\hline \multicolumn{8}{|l|}{$B M I$} \\
\hline Underweight & 9 & 17 & $2.03(0.88,4.69)$ & 18 & $2.37(1.02,5.47)$ & 14 & $1.67(0.68,4.08)$ \\
\hline Normal & 474 & 458 & 1.00 (reference) & 441 & 1.00 (reference) & 475 & 1.00 (reference) \\
\hline Overweight & 328 & 308 & $0.88(0.71,1.08)$ & 306 & $0.85(0.68,1.05)$ & 327 & $0.82(0.66,1.02)$ \\
\hline Obese & 89 & 81 & $0.79(0.56,1.10)$ & 67 & $0.61(0.43,0.87)$ & 63 & $0.52(0.36,0.76)$ \\
\hline $\mathrm{OR}_{\text {trend }}$ & & & $0.89(0.77,1.03)$ & & $0.81(0.70,0.95)$ & & $0.77(0.66,0.91)$ \\
\hline$P$ trend & & & 0.12 & & 0.009 & & 0.001 \\
\hline \multicolumn{8}{|l|}{ Family history } \\
\hline Absent & 820 & 799 & 1.00 (reference) & 767 & 1.00 (reference) & 822 & 1.00 (reference) \\
\hline Present & 80 & 65 & $0.88(0.62,1.25)$ & 65 & $1.00(0.70,1.43)$ & 57 & $0.87(0.59,1.27)$ \\
\hline P value & & & 0.47 & & 0.99 & & 0.46 \\
\hline
\end{tabular}

Odds ratios (ORs) and 95\% confidence intervals (CIs) were obtained using polytomous logistic regression models that were mutually adjusted for age, age at menarche, parity, body mass index (BMI) and family history of breast cancer in a first degree relative. Parity was adjusted in the main model (presented in table) and this was substituted for breastfeeding in a separate model. All models were further adjusted for year at diagnosis and the NPI score in complete case analysis. Overall, a total of 3475 subjects with complete information on IHC4, NPI, and breast cancer risk factors were included in the analysis presented in this table. In sensitivity analyses including missing value indicators for breast cancer risk factors and NPI ( $n=7685$; Supplementary Table 6), the results were similar to those presented here

determine whether previously reported associations between age at onset and recurrence or survival following breast cancer are mediated by the influence of age on specific tumor characteristics.
Our findings of associations between increasing parity and clinically advanced breast cancer is in line with previously reported associations between parity and aggressive breast cancer phenotypes [26-28]. Given that IHC markers 
Table 5 Odds ratios (ORs) and 95\% confidence intervals (CIs) for the associations between age, parity, body mass index (BMI), family history, and levels of the Nottingham prognostic index (NPI) and immunohistochemical 4 (IHC4) score among Polish breast cancer patients $(n=972)$

\begin{tabular}{|c|c|c|c|c|c|c|}
\hline \multirow[t]{3}{*}{ Characteristic } & \multicolumn{3}{|l|}{ NPI } & \multicolumn{3}{|l|}{ IHC4 } \\
\hline & Q2 & Q3 & Q4 & Q2 & Q3 & Q4 \\
\hline & $\mathrm{OR}(95 \% \mathrm{CI})$ & $\mathrm{OR}(95 \% \mathrm{CI})$ & $\mathrm{OR}(95 \% \mathrm{CI})$ & OR(95\% CI) & OR(95\% CI) & OR(95\% CI) \\
\hline \multicolumn{7}{|l|}{ Age, years } \\
\hline$<45$ & 1.00 (reference) & 1.00 (reference) & 1.00 (reference) & 1.00 (reference) & 1.00 (reference) & 1.00 (reference) \\
\hline $45-55$ & $0.74(0.39,1.41)$ & $0.77(0.41,1.47)$ & $0.72(0.37,1.37)$ & $1.16(0.57,2.35)$ & $0.69(0.36,1.34)$ & $0.63(0.32,1.24)$ \\
\hline $55-65$ & $0.59(0.30,1.14)$ & $0.60(0.31,1.18)$ & $0.45(0.22,0.89)$ & $1.64(0.78,3.44)$ & $0.90(0.45,1.83)$ & $0.70(0.34,1.45)$ \\
\hline$>65$ & $1.08(0.54,2.17)$ & $0.89(0.43,1.82)$ & $0.77(0.37,1.59)$ & $1.17(0.55,2.51)$ & $0.58(0.28,1.20)$ & $0.50(0.34,1.06)$ \\
\hline $\mathrm{OR}_{\text {trend }}$ & $1.05(0.85,1.28)$ & $0.96(0.78,1.19)$ & $0.91(0.74,1.14)$ & $1.04(0.84,1.29)$ & $0.89(0.72,1.11)$ & $0.84(0.67,1.06)$ \\
\hline$P$ trend & 0.67 & 0.74 & 0.43 & 0.68 & 0.30 & 0.14 \\
\hline \multicolumn{7}{|l|}{ Parity } \\
\hline Nulliparous & 1.00 (reference) & 1.00 (reference) & 1.00 (reference) & 1.00 (reference) & 1.00 (reference) & 1.00 (reference) \\
\hline 1 & $1.05(0.58,1.91)$ & $1.50(0.84,2.69)$ & $1.34(0.59,3.03)$ & $0.87(0.49,1.45)$ & $1.06(0.58,1.55)$ & $1.18(0.63,2.22)$ \\
\hline 2 & $0.79(0.45,1.38)$ & $0.80(0.46,1.41)$ & $0.86(0.38,1.93)$ & $0.69(0.39,1.22)$ & $1.04(0.58,1.87)$ & $1.07(0.58,1.99)$ \\
\hline$\geq 3$ & $0.72(0.41,1.29)$ & $0.82(0.46,1.45)$ & $1.05(0.47,2.36)$ & $1.20(0.55,2.63)$ & $0.86(0.36,2.05)$ & $1.33(0.56,3.15)$ \\
\hline $\mathrm{OR}_{\text {trend }}$ & $1.18(0.95,1.47)$ & $0.95(0.76,1.18)$ & $1.01(0.80,1.27)$ & $0.95(0.76,1.20)$ & $0.98(0.78,1.23)$ & $1.05(0.82,1.33)$ \\
\hline$P$ value & 0.14 & 0.63 & 0.96 & 0.69 & 0.87 & 0.71 \\
\hline \multicolumn{7}{|l|}{$B M I$} \\
\hline Underweight & $1.44(0.30,6.95)$ & $1.85(0.39,8.78)$ & $0.42(0.04,4.43)$ & $1.77(0.30,10.39)$ & $1.67(0.26,10.60)$ & $2.00(0.32,12.51)$ \\
\hline Normal & 1.00 (reference) & 1.00 (reference) & 1.00 (reference) & 1.00 (reference) & 1.00 (reference) & 1.00 (reference) \\
\hline Overweight & $1.00(0.63,1.58)$ & $1.27(0.80,2.03)$ & $1.34(0.82,2.19)$ & $1.08(0.67,1.73)$ & $0.95(0.58,1.54)$ & $0.82(0.49,1.36)$ \\
\hline Obese & $1.33(0.80,2.23)$ & $1.58(0.93,2.69)$ & $2.27(1.32,3.89)$ & $0.81(0.47,1.38)$ & $1.23(0.72,2.08)$ & $0.81(0.47,1.43)$ \\
\hline $\mathrm{OR}_{\text {trend }}$ & $1.13(0.88,1.46)$ & $1.24(0.96,1.61)$ & $1.48(1.13,1.93)$ & $0.92(0.70,1.19)$ & $1.11(0.85,1.45)$ & $0.89(0.67,1.06)$ \\
\hline$P$ trend & 0.34 & 0.10 & 0.004 & 0.51 & 0.43 & 0.44 \\
\hline \multicolumn{7}{|l|}{ Family history } \\
\hline Absent & 1.00 (reference) & 1.00 (reference) & 1.00 (reference) & 1.00 (reference) & 1.00 (reference) & 1.00 (reference) \\
\hline Present & $1.00(0.53,1.90)$ & $0.89(0.46,1.72)$ & $0.65(0.32,1.32)$ & $0.86(0.44,1.69)$ & $1.14(0.60,2.18)$ & $1.05(0.52,2.11)$ \\
\hline$P$ value & 0.99 & 0.73 & 0.24 & 0.67 & 0.68 & 0.89 \\
\hline
\end{tabular}

OR and 95\% CI were from polytomous logistic regression models (Q1 was the base (comparison) category) with mutual adjustments for age, parity, body mass index (BMI), age at menarche (not shown), and family history of breast cancer in a first degree relative. The NPI model was further adjusted for breast cancer subtype while the IHC4 model was adjusted for NPI

Table 6 Change in likelihood ratio Chi-square $\left(\Delta \mathrm{LR} \chi^{2}\right)$ and corresponding $\mathrm{p}$ values testing the contributions of individual clinicopathological characteristics to predictive models for body mass index (BMI), parity, and family history among Chinese breast cancer patients

\begin{tabular}{|c|c|c|c|c|c|c|c|c|}
\hline \multirow[t]{2}{*}{ Characteristic } & \multicolumn{2}{|c|}{ Overweight } & \multicolumn{2}{|l|}{ Obese } & \multicolumn{2}{|l|}{ Parous } & \multicolumn{2}{|c|}{ Positive FHBC } \\
\hline & $\Delta \mathrm{LR} \chi^{2}$ & P value & $\Delta \mathrm{LR} \chi^{2}$ & $P$ value & $\Delta \mathrm{LR} \chi^{2}$ & $P$ value & $\Delta \mathrm{LR} \chi^{2}$ & $P$ value \\
\hline Age, per year & 75.4 & $<0.001$ & 40.0 & $<0.001$ & 71.5 & $<0.001$ & 4.7 & 0.03 \\
\hline Tumor size, per $\mathrm{cm}$ & 4.0 & 0.04 & 10.7 & 0.001 & 2.3 & 0.13 & 0.3 & 0.29 \\
\hline Grade & 0.4 & 0.81 & 5.0 & 0.08 & 3.4 & 0.18 & 1.5 & 0.47 \\
\hline Node & 7.6 & 0.02 & 1.0 & 0.60 & 3.6 & 0.17 & 8.5 & 0.01 \\
\hline ER & 4.0 & 0.04 & 9.9 & 0.002 & 2.1 & 0.15 & 0.0 & 0.97 \\
\hline PR & 3.3 & 0.07 & 2.6 & 0.11 & 0.8 & 0.37 & 0.1 & 0.80 \\
\hline HER2 & 0.4 & 0.51 & 6.5 & 0.01 & 0.1 & 0.73 & 1.9 & 0.17 \\
\hline
\end{tabular}

All risk factors were mutually adjusted for one another in unconditional logistic regression models separately for overweight (vs normal weight), obesity (vs normal weight), parous (vs nulliparous), family history of breast cancer (vs no family history) as dependent variables and the clinicopathological characteristics as predictor variables. We determined the change in likelihood ratio Chi-square by removing each factor from the full predictive model and comparing the nested model with the full model using a likelihood ratio test 
and other tumor characteristics are highly correlated, it is unclear if previously reported associations between parity and HR- tumors are driven entirely by HR expression or by other clinicopathological characteristics. By accounting for many of these markers in the current analysis, we demonstrated that tumor characteristics contained in the NPI may partly explain previously observed associations between parity and HR- breast cancer. The mechanism through which parity predisposes to aggressive phenotypes of breast cancer is largely unknown but it is thought to be related, at least in part, to changes in the breast microenvironment that can result from aberrant post-partum lobular involution [29, 30]. Previous reports were in support of the attenuating effects of prolonged breastfeeding on the association between parity and TNBC, an aggressive subtype of breast cancer [27, 31, 32]. In contrast to these reports, however, we did not find evidence to support associations between breastfeeding and any of the prognostic parameters.

Results from epidemiological studies indicate that elevated BMI more strongly predisposes to HR- than HR + breast cancers among premenopausal women, with the converse predominating among postmenopausal women $[33,34]$. Given the superior survival indices for HR + over HR - breast cancer, some researchers have suggested that elevated BMI more strongly predisposes to less aggressive tumor subtypes, particularly in postmenopausal women [35]. Perhaps paradoxically, results from several other studies, including a large-scale pooling analysis, have supported higher frequencies of aggressive tumor characteristics, particularly histologic grade, among overweight and/or obese than normal weight women $[9,10,36]$. Our findings that elevated BMI was associated with low IHC4 on the one hand, and high NPI on the other hand, might explain previous reports since low IHC4 corresponds to high HR expression whereas high NPI can connote larger tumor size, higher histologic grade and/or positive lymph nodal status, all markers of poor prognosis. Notably, the associations between BMI and NPI were irrespective of HR expression, suggesting that overweight and/or obese women were more likely to develop aggressive tumor subtypes regardless of HR expression status. Our observation that several tumor characteristics were statistically significantly predictive of overweight and obese BMI following LR tests indicates that the somewhat paradoxical relationship between BMI and tumor aggressiveness might be due to varying roles of estrogen metabolism, adiponectin, insulin-like growth factors, chronic inflammation, and/or delayed detection in obesity-related breast carcinogenesis [29, 37-39].

The literature on the relationship between FHBC and clinical outcomes in breast cancer is not consistent [40-44]. In the current study, we observed having a positive FHBC to be associated with lower NPI values, suggesting better prognosis. In analysis assessing the contributions of the individual tumor characteristics to a predictive model for FHBC, we found lymph nodal status to provide more predictive information than other tumor characteristics. This might suggest that women with a positive FHBC were less likely to have clinically advanced disease than those without a FHBC. A possible explanation for this finding may be that women with a positive FHBC may be more likely to seek and/or undergo screening or other forms of surveillance which can lead to the detection of early stage tumors than those without a FHBC. This may be especially true in countries where large-scale, organized, breast cancer screening is not available and where selective screening is offered to high risk women (partly defined by having a positive FHBC). Accordingly, further studies with detailed screening histories will be required to conclusively determine relationships between FHBC, prognostic biomarkers, and clinical outcomes in breast cancer.

We assessed the external generalizability of findings from the Chinese population by investigating the key results in an independent population of Polish breast cancer patients. With the exception of parity, the associations between risk factors and NPI were in the same direction in the Polish and Chinese populations. In particular, the associations between BMI and NPI were significant in both populations. Unlike Chinese patients, for whom increasing parity was statistically significantly associated with higher NPI, parity was not associated with higher NPI among the Polish patients. Given the documented associations between pregnancy-associated breast cancer, which could occur up to 10 years after pregnancy, and aggressive phenotypes of breast cancer [45], it is possible that differences in results between the study populations may reflect differences in time since last childbirth. We were unable to specifically evaluate this question due to lack of information on time since last birth and breast cancer diagnosis in both study populations. Nonetheless, Polish participants were older and more likely to be postmenopausal than the Chinese participants which might suggest a longer time since last birth for the Polish patients. Future studies will be warranted to investigate the impact of time since last birth on clinically relevant prognostic biomarkers in breast cancer.

An important consideration for the development of targeted prevention strategies in breast cancer is the identification of women at high risk of developing the most aggressive subtypes of breast cancer, for whom interventions to prevent invasive disease can be instituted. Based on our findings, higher parity as well as being overweight or obese were associated with poor prognostic indices, suggesting a potential beneficial effect for improved surveillance among such women. Relatedly, these findings may be suggestive of the importance of including breast cancer risk factors as part of breast cancer prognostic models. To date, only PREDICT has included breast cancer risk factors, i.e. age at 
diagnosis and mode of detection, in its calculations and has been shown to outperform the IHC4 score, IHC subtyping, and C-score in terms of prognostic power [46]. It is probable that future models incorporating BMI may provide further survival discrimination in breast cancer patients beyond what is contained in existing models.

The assembly of several clinicopathological and epidemiological factors for $>8000$ patients is an important strength of this study. In terms of limitations, however, this population is largely unscreened, as such, some of the reported associations may be subject to detection bias. For example, smaller tumors may be less likely to be palpated in obese than normal weight women. Accordingly, overweight and/ or obese women may be more likely to have larger tumors on the basis of detection alone. Nevertheless, other tumor characteristics were independently associated with higher BMI in this study, suggesting some biological underpinning for our findings. Moreover, detection bias is itself a clinically important problem that needs to be addressed. Also, we did not have clinical outcome data such as recurrence or survival for these patients, which precluded our ability to perform mediation analyses or to directly examine associations between breast cancer risk factors and clinical outcomes. Nonetheless, NPI and IHC4 score strongly predict clinical outcomes in breast cancer. Together, they reflect the spectrum of clinicopathological characteristics that are used in routine clinical practice to inform breast cancer management.

\section{Conclusions}

In conclusion, our results support associations between breast cancer risk factors and two clinically useful prognostic factors in breast cancer, i.e. NPI and IHC4. Specifically, we found younger age at onset, higher parity, being overweight/obese and absent FHBC to be associated with poor prognostic indices, indicated by higher NPI score, irrespective of HR expression. Although elevated BMI was associated with low IHC4 score, connoting clinically less aggressive HR + tumors, NPI-related findings were supportive of poor prognosis among overweight/obese patients irrespective of tumor HR status. These findings highlight important relationships between breast cancer risk factors and clinically relevant prognostic factors, with potential implications for the development of surveillance, prognostication, and counseling strategies that take host factors into account.

Supplementary Information The online version contains supplementary material available at https://doi.org/10.1007/s10549-021-06294-5.

Acknowledgements We acknowledge Drs. Jonine D. Figueroa, Thomas U. Ahearn and Jolanta Lissowska for providing data from the PBCS for replication analyses.
Author contributions Conception and design: MA, NL, XRY. Development of methodology: MA, CG, NL, XRY. Acquisition and management of data: CG, HK, NH, BZ, NL, MGC. Analysis and interpretation of data: MA, MGC, XRY. Writing, review and/or revision of the manuscript: MA, CG, HK, BZ, JD, NH, BZ, MGC, NL, XRY. Study supervision: XRY.

Funding This research was supported by the Intramural Research Program of the National Institutes of Health, National Cancer Institute, Division of Cancer Epidemiology and Genetics.

Data availability All the datasets used and/or analyzed during the current study are available from the corresponding author on reasonable request.

Code availability Not applicable.

\section{Declarations}

Conflict of interest The authors declare that they have no competing interests.

Ethical approval This project received ethical approval from the CHCAMS Ethics Committee and was exempted from review by the Office of Human Research Protections at the National Institutes of Health, NIH (exempt number 11751), since it did not involve interaction with human subjects and/or use of individual's personal identifying information. Informed consent was not required for the use of existing pathological materials with no reveal of identifiable patient information.

Consent to participate Not applicable.

Consent for publication Not applicable.

Open Access This article is licensed under a Creative Commons Attribution 4.0 International License, which permits use, sharing, adaptation, distribution and reproduction in any medium or format, as long as you give appropriate credit to the original author(s) and the source, provide a link to the Creative Commons licence, and indicate if changes were made. The images or other third party material in this article are included in the article's Creative Commons licence, unless indicated otherwise in a credit line to the material. If material is not included in the article's Creative Commons licence and your intended use is not permitted by statutory regulation or exceeds the permitted use, you will need to obtain permission directly from the copyright holder. To view a copy of this licence, visit http://creativecommons.org/licenses/by/4.0/.

\section{References}

1. Goldhirsch A, Wood WC, Coates AS, Gelber RD, Thürlimann B, Senn H-J et al (2011) Strategies for subtypes-dealing with the diversity of breast cancer: highlights of the St Gallen International Expert Consensus on the Primary Therapy of Early Breast Cancer 2011. Ann Oncol 22(8):12

2. Coates AS, Members P, Winer EP, Members P, Goldhirsch A, Members $P$ et al (2015) Tailoring therapies-improving the management of early breast cancer: St Gallen International Expert Consensus on the Primary Therapy of Early Breast Cancer 2015. Ann Oncol 26(8):14 
3. Caleffi M, Fentiman IS, Birkhead BG (1989) Factors at presentation influencing the prognosis in breast cancer. Eur J Cancer Clin Oncol 25(1):51-56

4. Trivers KF, Gammon MD, Abrahamson PE, Lund MJ, Flagg EW, Kaufman JS et al (2007) Association between reproductive factors and breast cancer survival in younger women. Breast Cancer Res Treat 103(1):93-102

5. Alsaker MDK, Opdahl S, Asvold BO, Romundstad PR, Vatten LJ (2011) The association of reproductive factors and breastfeeding with long term survival from breast cancer. Breast Cancer Res Treat 130(1):175-182

6. Kwan ML, Bernard PS, Kroenke CH, Factor RE, Habel LA, Weltzien EK et al (2015) Breastfeeding, PAM50 tumor subtype, and breast cancer prognosis and survival. JNCI 107(7):087

7. Cespedes Feliciano EM, Kwan ML, Kushi LH, Chen WY, Weltzien EK, Castillo AL et al (2017) Body mass index, PAM50 subtype, recurrence, and survival among patients with nonmetastatic breast cancer. Cancer 123(13):2535-2542

8. Abubakar M, Sung H, Devi BCR, Guida J, Tang TS, Pfeiffer RM et al (2018) Breast cancer risk factors, survival and recurrence, and tumor molecular subtype: analysis of 3012 women from an indigenous Asian population. Breast Cancer Res. 20(1):djv114

9. Borgquist S, Wirfält E, Jirström K, Anagnostaki L, Gullberg B, Berglund G et al (2007) Diet and body constitution in relation to subgroups of breast cancer defined by tumour grade, proliferation and key cell cycle regulators. Breast Cancer Res. 9(1):R11

10. Santa-Maria CA, Yan J, Xie X-J, Euhus DM (2015) Aggressive estrogen-receptor-positive breast cancer arising in patients with elevated body mass index. Int J Clin Oncol 20(2):317-323

11. Garcia-Closas M, Brinton L, Lissowska J, Chatterjee N, Peplonska B, Anderson WF et al (2006) Established breast cancer risk factors by clinically important tumour characteristics. British J Cancer 95(1): 123-129

12. Mustapha A, Jenny C-C, Raza AH, Nilanjan C, Penny C, Frances D et al (2018) Etiology of hormone receptor positive breast cancer differs by levels of histologic grade and proliferation. Int J Cancer 143(4):746-757

13. Galea MH, Blamey RW, Elston CE, Ellis IO (1992) The Nottingham prognostic index in primary breast cancer. Breast Cancer Res Treat 22(3):207-219

14. Wishart GC, Bajdik CD, Dicks E, Provenzano E, Schmidt MK, Sherman M et al (2012) PREDICT Plus: development and validation of a prognostic model for early breast cancer that includes HER2. Br J Cancer 107(5):800-807

15. Klein ME, Dabbs DJ, Shuai Y, Brufsky AM, Jankowitz R, Puhalla SL et al (2013) Prediction of the oncotype DX recurrence score: use of pathology-generated equations derived by linear regression analysis. Mod Pathol 26(5):658-664

16. Cuzick J, Dowsett M, Pineda S, Wale C, Salter J, Quinn E et al (2011) Prognostic value of a combined estrogen receptor, progesterone receptor, $\mathrm{Ki}-67$, and human epidermal growth factor receptor 2 immunohistochemical score and comparison with the Genomic Health recurrence score in early breast cancer. J Clin Oncol 29(32):4273-4278

17. Yang XR, Sherman ME, Rimm DL, Lissowska J, Brinton LA, Peplonska B et al (2007) Differences in risk factors for breast cancer molecular subtypes in a population-based study. Cancer Epidemiol Biomark Prev 16(3):439-443

18. Abubakar M, Guo C, Koka H, Sung H, Shao N, Guida J et al (2019) Clinicopathological and epidemiological significance of breast cancer subtype reclassification based on p53 immunohistochemical expression. npj Breast Cancer. 5(1):20

19. Petrelli F, Viale G, Cabiddu M, Barni S (2015) Prognostic value of different cut-off levels of Ki-67 in breast cancer: a systematic review and meta-analysis of 64,196 patients. Breast Cancer Res Treat 153(3):477-491
20. Goldhirsch A, Winer EP, Coates AS, Gelber RD, Piccart-Gebhart M, Thürlimann B et al (2013) Personalizing the treatment of women with early breast cancer: highlights of the St Gallen International Expert Consensus on the Primary Therapy of Early Breast Cancer 2013. Ann Oncol 24(9):18

21. El Saghir NS, Seoud M, Khalil MK, Charafeddine M, Salem ZK, Geara FB et al (2006) Effects of young age at presentation on survival in breast cancer. BMC Cancer 6(1):194

22. Fredholm H, Eaker S, Frisell J, Holmberg L, Fredriksson I, Lindman H (2009) Breast cancer in young women: poor survival despite intensive treatment. PloS One. 4(11):e7695

23. Anders CK, Hsu DS, Broadwater G, Acharya CR, Foekens JA, Zhang Y et al (2008) Young age at diagnosis correlates with worse prognosis and defines a subset of breast cancers with shared patterns of gene expression. J Clin Oncol 26(20):3324-3330

24. Anders CK, Johnson R, Litton J, Phillips M, Bleyer A (2009) Breast cancer before age 40 years. Semin Oncol 36(3):237-249

25. Anderson WF, Chen BE, Brinton LA, Devesa SS (2007) Qualitative age interactions (or effect modification) suggest different cancer pathways for early-onset and late-onset breast cancers. Cancer Causes Control 18(10):1187-1198

26. Palmer JR, Boggs DA, Wise LA, Ambrosone CB, Adams-Campbell LL, Rosenberg L (2011) Parity and lactation in relation to estrogen receptor negative breast cancer in African American Women. Cancer Epidemiol Biomark Prev 20(9):1883-1891

27. Palmer JR, Viscidi E, Troester MA, Hong C-C, Schedin P, Bethea TN et al (2014) Parity, lactation, and breast cancer subtypes in african american women: results from the AMBER consortium. JNCI. 106(10):dju37

28. Dietze EC, Sistrunk C, Miranda-Carboni G, O’Regan R, Seewaldt VL (2015) Triple-negative breast cancer in African-American women: disparities versus biology. Nat Rev Cancer 15(4):248-254

29. Baumgarten SC, Frasor J (2012) Minireview: inflammation: an instigator of more aggressive estrogen receptor (ER) positive breast cancers. Mol Endocrinol 26(3):360-371

30. Ruiz R, Herrero C, Strasser-Weippl K, Touya D, St Louis J, Bukowski A et al (2017) Epidemiology and pathophysiology of pregnancy-associated breast cancer: a review. Breast (Edinburgh, Scotland) 35:136-141

31. Ambrosone CB, Zirpoli G, Ruszczyk M, Shankar J, Hong C-C, McIlwain D et al (2014) Parity and breastfeeding among AfricanAmerican women: differential effects on breast cancer risk by estrogen receptor status in the Women's Circle of Health Study. Cancer Causes Control 25(2):259-265

32. Chen L, Li CI, Tang M-TC, Porter P, Hill DA, Wiggins CL et al (2016) Reproductive factors and risk of luminal, HER2-overexpressing, and triple-negative breast cancer among multiethnic women. Cancer Epidemiol Biomarkers Prevent 25(9):1297-1304

33. Kwan ML, Kushi LH, Weltzien E, Maring B, Kutner SE, Fulton RS et al (2009) Epidemiology of breast cancer subtypes in two prospective cohort studies of breast cancer survivors. Breast Cancer Res 11(3):1-13

34. Yang XR, Chang-Claude J, Goode EL, Couch FJ, Nevanlinna $\mathrm{H}$, Milne RL et al (2011) Associations of breast cancer risk factors with tumor subtypes: a pooled analysis from the Breast Cancer Association Consortium studies. J Natl Cancer Inst 103(3):250-263

35. Nattenmüller CJ, Kriegsmann M, Sookthai D, Fortner RT, Steffen A, Walter B et al (2018) Obesity as risk factor for subtypes of breast cancer: results from a prospective cohort study. BMC Cancer 18(1):616

36. Yanai A, Miyagawa Y, Murase K, Imamura M, Yagi T, Ichii S et al (2014) Influence of body mass index on clinicopathological factors including estrogen receptor, progesterone receptor, 
and Ki67 expression levels in breast cancers. Int J Clin Oncol 19(3):467-472

37. Iyengar NM, Hudis CA, Dannenberg AJ (2013) Obesity and inflammation: new insights into breast cancer development and progression. Am Soc Clin Oncol Educational Book/ASCO Am Soc Clin Oncol Meeting 33:46-51

38. Agresti R, Meneghini E, Baili P, Minicozzi P, Turco A, Cavallo I et al (2016) Association of adiposity, dysmetabolisms, and inflammation with aggressive breast cancer subtypes: a cross-sectional study. Breast Cancer Res Treat 157(1):179-189

39. Dietze EC, Chavez TA, Seewaldt VL (2018) Obesity and triplenegative breast cancer: disparities, controversies, and biology. Am J Pathol 188(2):280-290

40. Welsh ML, Buist DSM, Aiello Bowles EJ, Anderson ML, Elmore JG, Li CI (2009) Population-based estimates of the relation between breast cancer risk, tumor subtype, and family history. Breast Cancer Res Treat 114(3):549-558

41. Zhou W, Pan H, Liang M, Xia K, Liang X, Xue J et al (2013) Family history and risk of ductal carcinoma in situ and triple negative breast cancer in a Han Chinese population: a case-control study. World Journal of Surgical Oncology 11(1):248

42. Jiang X, Castelao JE, Chavez-Uribe E, Fernandez Rodriguez B, Celeiro Muñoz C, Redondo CM et al (2012) Family history and breast cancer hormone receptor status in a Spanish cohort. PloS One. 7(1):e29459
43. Mavaddat N, Pharoah PD, Blows F, Driver KE, Provenzano E, Thompson D et al (2010) Familial relative risks for breast cancer by pathological subtype: a population-based cohort study. Breast Cancer Res 12(1):R10

44. Malone KE, Daling JR, Doody DR, O'Brien C, Resler A, Ostrander EA et al (2011) Family history of breast cancer in relation to tumor characteristics and mortality in a population-based study of young women with invasive breast cancer. Cancer Epidemiol Biomark Prev 20(12):2560-2571

45. Schedin P (2006) Pregnancy-associated breast cancer and metastasis. Nat Rev Cancer 6(4):281-291

46. Abubakar M, Figueroa J, Ali HR, Blows F, Lissowska J, Caldas C et al (2019) Combined quantitative measures of ER, PR, HER2, and KI67 provide more prognostic information than categorical combinations in luminal breast cancer. Mod Pathol 32(9):1244-1256

Publisher's Note Springer Nature remains neutral with regard to jurisdictional claims in published maps and institutional affiliations. 